\title{
Non-compact Groups, Coherent States, Relativistic Wave Equations and the Harmonic Oscillator
}

\author{
Diego Julio Cirilo-Lombardo
}

Published online: 21 November 2007

(C) Springer Science+Business Media, LLC 2007

\section{Erratum to: Found Phys}

DOI 10.1007/s10701-007-9110-3

Dear Reader,

Unfortunately the paper by Prof. Diego Julio Cirilo-Lombardo, "Non-compact Groups, Coherent States, Relativistic Wave Equations and the Harmonic Oscillator" which was published in Foundations of Physics, has been published twice.

The paper, in a correct final form, has been published in Foundations of Physics, volume 37, issue 6, June 2007, pp 919-950.

An earlier incorrect version of the paper has been published by mistake in Foundations of Physics, volume 37, issue 8, August 2007, pp 1149-1180. Please do not use this paper for citations or any other purpose.

We sincerely apologize for this mistake and for any inconvenience this may have caused.

\author{
Sonja Japenga \\ Publishing Editor 'Foundations of Physics' \\ Sonja.japenga@springer.com
}

The online version of the original article can be found at dx.doi.org/10.1007/s10701-007-9110-3.

D.J. Cirilo-Lombardo $(\bowtie)$

Bogoliubov Laboratory of Theoretical Physics and Joint Institute for Nuclear Research,

141980, Dubna, Russia

e-mail:diego@thsunl.jinr.ru 